Note

\title{
Quantitative and Qualitative Evaluation of Fatty Acids in Coffee Oil and Coffee Residue
}

\author{
Yuri Koshima ${ }^{1}$, Yutaka Kitamura ${ }^{2 *}$, M.Z. IsLAm $^{1}$ and Mito KoKawA ${ }^{2}$ \\ ${ }^{I}$ Graduate School of Life and Environmental Sciences, University of Tsukuba, 1-1-1, Tennodai, Tsukuba, Ibaraki 305- \\ 8572, Japan \\ ${ }^{2}$ Faculty of Life and Environmental Sciences, University of Tsukuba, 1-1-1, Tennodai, Tsukuba, Ibaraki 305-8572, \\ Japan
}

Received November 26, 2019 ; Accepted March 2, 2020

This study aimed to investigate the effects of roasting conditions and brewing on coffee oil quality and quantity to gain a basic understanding of coffee residue utilization. The fatty acid composition of the coffee oil extracted from roasted coffee bean and residue was analyzed. The optimum extraction time of whole coffee oil was six-hours using the Soxhlet method with hexane as a solvent. Roasting conditions influenced the extraction ratio of coffee oil, and the extraction ratio increased from 9.8 to $15.9 \%$ when the roasting level was increased from light roast $\left(211{ }^{\circ} \mathrm{C}\right.$ for $\left.10 \mathrm{~min}\right)$ to dark roast $\left(230{ }^{\circ} \mathrm{C}\right.$ for $\left.12 \mathrm{~min}\right)$. On the other hand, the ratio of extracted oil from the coffee residue after brewing was $5.9 \%$, which was $50 \%$ of the total coffee oil before brewing. The fatty acid composition of coffee oil was determined by GC/FID, and results indicated that the fatty acid composition between different roasting conditions and coffee residue did not differ significantly. The primary fatty acids were found in all samples and consisted of myristic (C14:0), palmitic (C16:0), stearic (C18:0), oleic (C18:1), linoleic (C18:2), linolenic (C18:3), and arachidic (C20:0) acid.

Keywords: coffee oil, fatty acids, GC/FID, oil extraction, coffee residue, particle size analysis

\section{Introduction}

Coffee is one of the fastest growing beverages consumed in the world. About 9.3 million tons of coffee was consumed in 2016 , with about 8.5 million tons $(90 \%)$ of the consumed coffee discarded as a residue (Budryn et al., 2012). Most of the produced coffee is harvested, roasted, and brewed for human consumption. One of the key components of the coffee bean is the coffee oil. Coffee oil is known to have abundant volatile compounds such as aldehydes, ketones, furans, pyrroles, pyrazines, pyridines and phenolic compounds (Calligaris et al., 2009). Lipids in the coffee bean consist of $75 \%$ triacylglycerol (TAG) and $18 \%$ diterpene alcohols and fatty acids. Most of the fatty acids in brewed coffee exist as free esters. (Speer and Kölling-Speer, 2006; Rendón, De Jesus Garcia Salva, and Bragagnolo, 2014). Fatty acids are considered to be an accurate indicator of coffee quality evaluation. The composition of fatty acids depends on various factors such as varieties and production areas, and comparison of fatty acid patterns is considered to be an effective method for classifying coffee (Speer and Kölling-Speer, 2006, Martín et al., 2001, Budryn et al., 2012).

The processing of coffee beans involves chemical and physical changes. Especially during roasting, many chemical changes occurring under high temperature and pressure greatly contribute to the formation of unique coffee flavors (Giacalone et al., 2019). The chemical reactions that occurs during roasting alters about $30-40 \%$ of the internal coffee bean components, including the aroma component; however, about $60-70 \%$ of the coffee bean substances, such as lipids and fibers, are chemically stable (Tanbe, 2016). On the other hand, physical changes include evaporation of water, volatilization of internal components, mixing, and destruction of cell walls also occur. 
As a result, bean weight is reduced by $10-20 \%$ due to roasting (Jokanović et al., 2012). Coffee oil is one of the chemically stable substances in coffee beans. In green coffee beans, coffee oil is suspended in the cytoplasm as oil droplets. During roasting, this oil dissolves volatile components and gases generated by roasting and protects them from heat degradation (De Oliveira et al., 2005; T Yukihiro, 2016). Since coffee oil has a unique flavor and nutritional properties, it could be used in food formulations. (Calligaris et al., 2009).

Before coffee is extracted as a beverage, part of the coffee oil adheres to the surface of the beans and others are present inside the beans. During extraction, the fluidity of the oil on the powder surface increases due the increased temperature and is exfoliated and eluted into the liquid, while the oil present inside the coffee grounds is less likely to be exfoliated. Thus, after extraction, part of the coffee oil remains in the residue and is discarded (Tanbe, 2016). During paper filtration, lipids remain mainly in the residue, and the paper filter showed little oil retention (Ratnayake et al., 1993). The coffee residue contains a large number of crude components such as crude protein, crude lipid, and dietary fiber (Mussatto et al., 2011b). The effective utilization of coffee residue has not been established due to its high level of material heterogeneity, high water content and decomposed components (De Oliveira et al., 2005). Previous studies have attempted several productive uses of coffee residues. Solutions have been considered for use in the food industry by extracting sugar, and as a biofuel by extracting fats and oils (Microbiol et al., 2000; Mussatto et al., 2011a; Efthymiopoulos et al., 2019). However, the industrial application of these methods has not yet been established. As coffee consumption continues to increase, it is considered necessary to study effective uses of the coffee residue. Establishing new uses to add value to coffee residues would lead to environmental and economic benefits.

The present study aimed to obtain basic knowledge for the effective use of coffee oil in coffee residues. It focuses on investigating the characteristics of coffee oils through the analysis of fatty acids. The particle size, water content of the residue, and the fatty acid of the coffee beans, which were roasted at three different levels, were analyzed to clarify the extraction characteristics and fatty acid composition of coffee oil in roasted beans and residue.

\section{Materials and Methods}

Materials and reagents Green coffee beans (Coffea Arabica) harvested in Nyeri, Kenya, in October 2017 were provided from SAZA Coffee Co., Ltd. (Ibaraki, Japan). For GC/FID analysis, the following standards were used: methyl myristate, methyl palmitate, methyl palmitoleate, methyl oleate, and cis-11-methyl eicosenoate from Wako Pure Chemical Industries (Osaka, Japan), and methyl stearate, methyl linoleate, methyl linolenate, methyl arachidate, methyl behenate, and methyl lignocerinate from Sigma Aldrich Japan
(Tokyo, Japan). Hexane from Wako Pure Chemical Industries (Osaka, Japan) was used as the solvent for lipid extraction.

Sample preparation Green coffee beans were roasted in 3 different conditions, namely light, medium, and dark roasts. The green coffee beans (1 $200 \mathrm{~g}$ per batch) were roasted using a coffee roaster (Probatone, Probat, Germany), and the temperature and duration of the roasts were $211^{\circ} \mathrm{C}$ for $11 \mathrm{~min}$, $215^{\circ} \mathrm{C}$ for $12 \mathrm{~min}$, and $230^{\circ} \mathrm{C}$ for $12 \mathrm{~min} 30 \mathrm{~s}$, for the light, medium, and dark roasts, respectively. The roasting temperatures and time were determined by a highly trained roaster based on the sensory properties of the coffee and considering the SCA (Specialty Coffee Association) international standards. After the roasting process, coffee beans were removed from the roaster and cooled until reaching room temperature. Roasted beans were packed in storage bags made by PET, aluminum and polyethylene film and kept until analysis.

A total of $60 \mathrm{~g}$ of ground coffee was prepared for the sieving experiment by grinding the medium roasted bean in three batches ( $20 \mathrm{~g}$ for each batch) with a coffee grinder (Next G, Kalita, U.S.). For oil extraction experiments, light, medium, and dark roasted coffee beans were ground and weighted to $20 \mathrm{~g}$.

For the preparation of the residue sample, $20 \mathrm{~g}$ of the medium roasted grounded coffee bean was brewed using $300 \mathrm{~mL}$ of hot water $\left(85^{\circ} \mathrm{C}\right)$ with a coffee maker with a metal filter (NC-R500, Panasonic). After brewing, the coffee residue was dried at room temperature for 30-60 min and immediately utilized in subsequent experiments.

Particle size analysis The particle size distribution of ground samples (medium roast) was measured using a sieve shaker with ten screens $(125-1572 \mu \mathrm{m})$ (TANAKA TEC CO., Japan). Sixty grams of ground samples were shaken for $7 \mathrm{~min}$. The size distribution was determined from the measured particle size and weight of the ground coffee. Each sample was analyzed five times.

Lipid extraction For lipid extraction, $20 \mathrm{~g}$ of ground coffee (light, medium, dark, and residue) was eluted with $300 \mathrm{~mL}$ of hexane for 1, 2, 3, 4, 5, 6 and $8 \mathrm{~h}$ to analyze the extraction efficiency by the Soxhlet method. The extract was then collected and concentrated at $40{ }^{\circ} \mathrm{C}$ under a vacuum for $30 \mathrm{~min}$ in a rotary evaporator (N-1100S, Tokyo Rikakikai CO). The extraction ratio was calculated as a percentage of oil weight $(\mathrm{g}) /$ bean weight $(\mathrm{g})$. Each analysis was done in triplicate. To evaluate the lipid content of coffee residue on a dry basis, the moisture content of the residue sample was measured in triplicate.

Fatty acid analysis by GC/FID The extracts of the light, medium, dark roast coffee, and residue samples were methylesterified using a methyl esterification kit (Shinwa DS-TG, Shinwa Chemical Co., Ltd.). All samples were analyzed by gas chromatography with a flame ionization detector (GC/FID) according to the method described in a previous study (Martín 

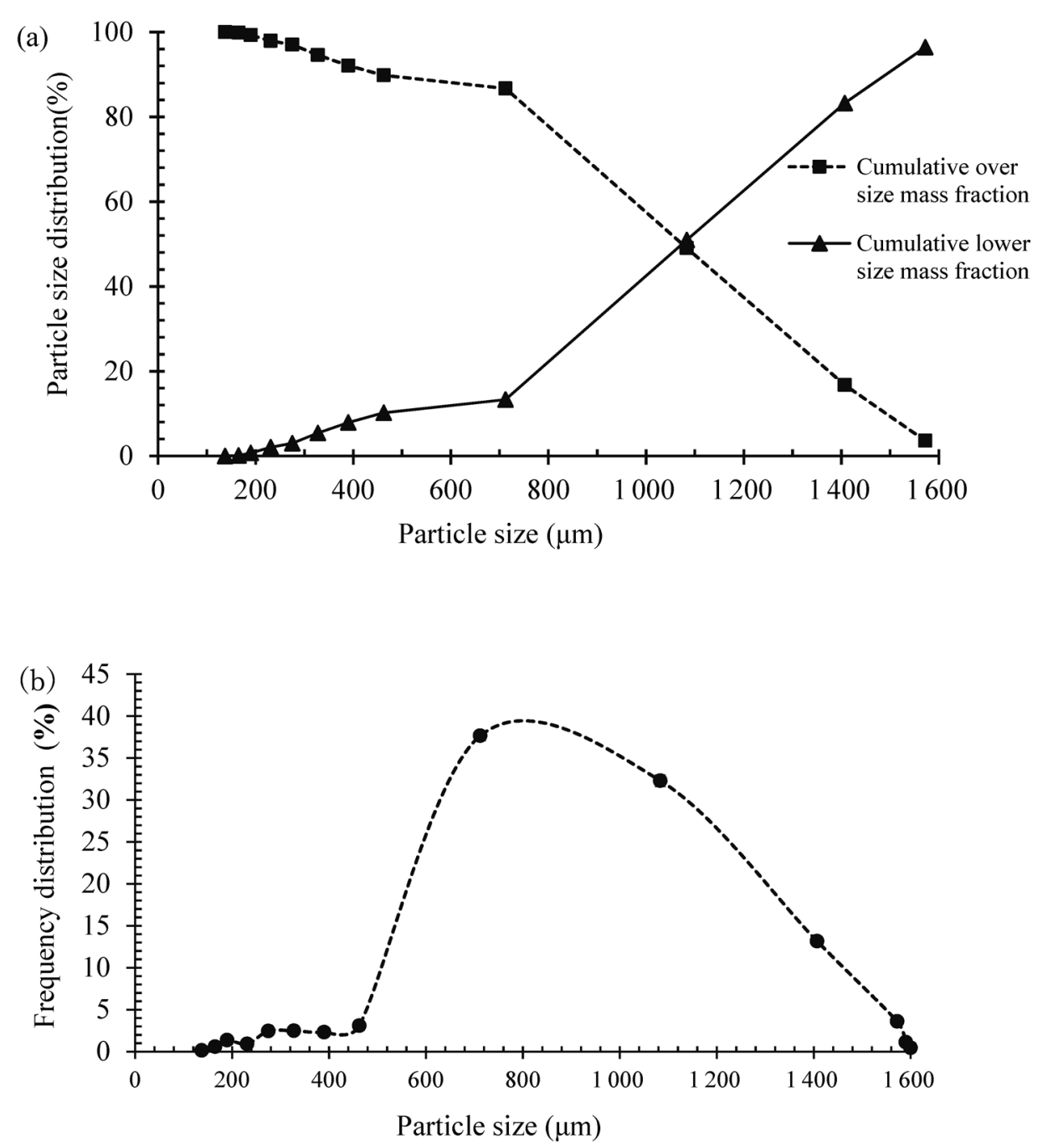

Fig. 1. (a) Mass fraction and (b) Frequency on particle distribution of ground coffee bean (medium roast).

The data shows the average values of the five samples.

et al., 2001). Qualification and quantification were performed using the external standard method (Martín et al., 2001). Eleven fatty acid methyl ester (FAME) standards, namely, myristic (C14:0), palmitic (C16:0), palmitoleic (C16:1), stearic (C18:0), oleic (C18:1), linoleic (C18:2), linolenic (C18:3), arachidic (C20:0), eicosenoic, behenic, and lignoceric acids, were utilized for qualitative and quantitative analysis. The sample concentrations were adjusted with $n$-hexane. The sample solution $(1 \mu \mathrm{L})$ was injected by an autosampler to the gas chromatograph (GC-14B, Shimazu) with a Shinchrom E71 packed column $(3.1 \mathrm{~m} \times 3.2 \mathrm{~mm} \mathrm{I.D)}$, and the carrier gas was nitrogen with a flow rate of $50 \mathrm{~mL} / \mathrm{min}$. The injection, column, and detection temperatures were 260,210 , and $260{ }^{\circ} \mathrm{C}$, respectively.

Statistical analysis Statistical analysis was performed using JMP 13.0.0 (SAS Institute Inc., Carolina, U.S.). Tukey's test and $t$-tests were used to determine the significant differences in fatty acid components $(p<0.05)$ and the effects of roasting and brewing.

\section{Results and Discussion}

Particle size of ground coffee beans The particle size of the ground coffee beans and the extraction characteristics are closely related. Finer particle sizes increase the surface area where the solvent and the coffee particles come in contact and finally enhance the extraction of lipids. The lipids are deposited on the surface of the ground coffee and contain many color and flavor components. Generally, the smaller the particle size, the more the flavor components are extracted (Tanbe, 2016). In the present study, a cutting type grinder was used to obtain samples with uniform particle size. The particle size distribution and the cumulative distribution curve for the medium roasted coffee obtained by the screening test are shown in Fig. 1a and $1 \mathrm{~b}$. Average values of five replicate measurements are shown. The mean particle size of the ground beans was $712 \mu \mathrm{m}$, and the median diameter was $1083 \mu \mathrm{m}$. The particle size of the roasted coffee bean was more or less similar to the results reported by Maruo and Hirose (2009), who mentioned that the particle size of ground coffee commonly used for coffee beverage extraction 

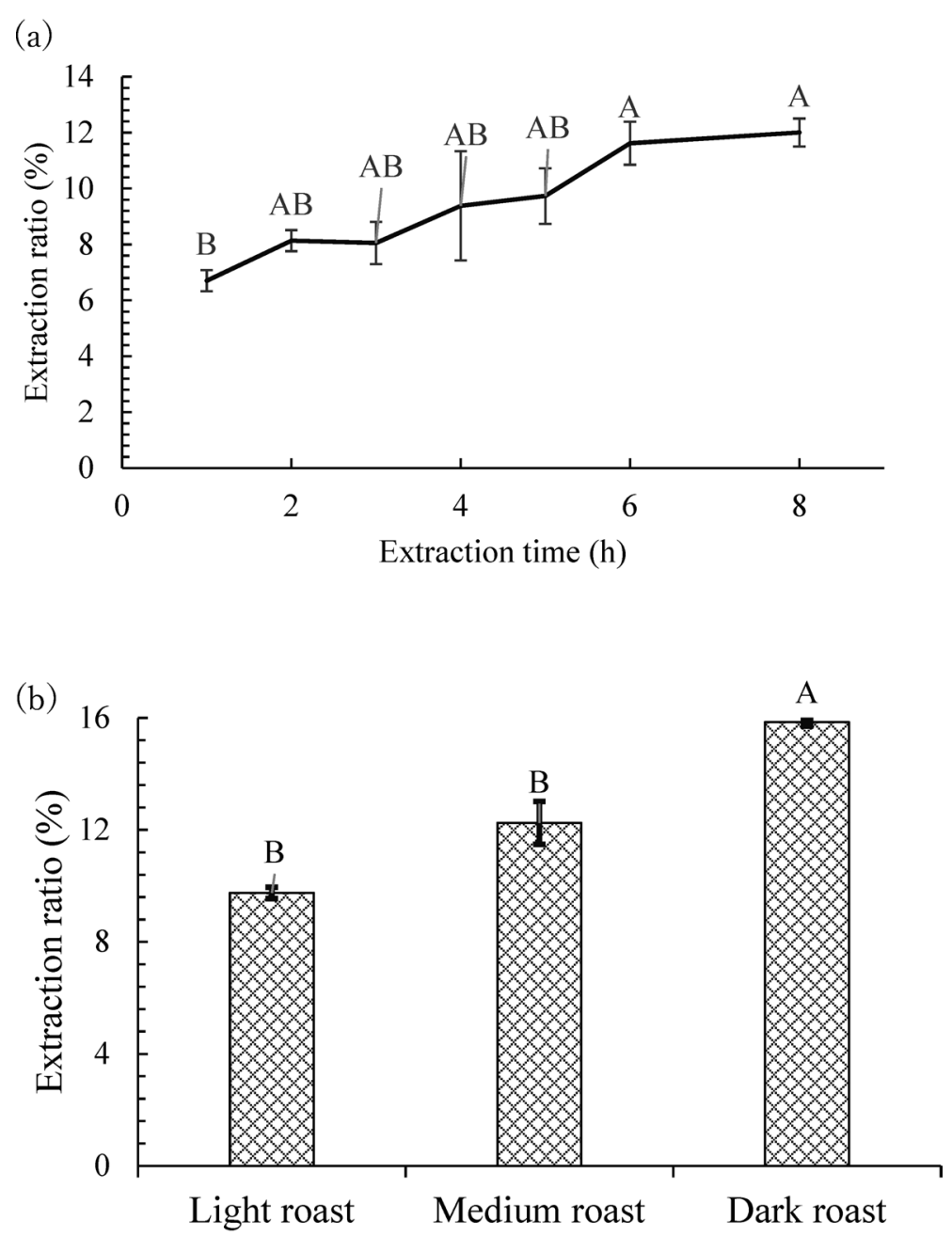

Fig. 2. (a) The relationship between the extraction time and the oil extraction ratio and (b) effect of roasting on oil extraction from coffee bean

(medium grind) ranged from 600 to $1000 \mu \mathrm{m}$. The samples used in this study showed more uniform particles with less variation in particle size.

Effect of roasting on lipid extraction The relationship between extraction time and lipid extraction ratio (oil weight/ bean weight) of medium roasted coffee samples is shown in Fig. 2a. The extraction ratio gradually increased with prolonged extraction time and plateaued after $6 \mathrm{~h}$. No significant difference was found between extraction ratios at 6 and $8 \mathrm{~h}$. The maximum extraction ratio obtained was $12 \%$, which is close to the total amount of lipid contained in the coffee bean (R.J. Clarke and Macrae, 1985). Our results are in accordance with the findings presented by Alves et al. (2003), who reported that lipid content in roasted ground coffee beans was in the range of 8 to $15 \%$.

The oil extraction ratios of the three roasted coffee samples (light, medium, and dark roasts) are shown in Fig. 2b. The results indicate that roasting conditions significantly influence the extraction ratio, and when the roasting conditions are changed from light to dark, the extraction ratio increases from
9.8 to $15.9 \%$. This is thought to be mainly due to dark roasted coffee having a higher percentage of solids and oils due to moisture evaporation during the roasting process. Similarly, a previous study reported that when the roasting temperature increased from 190 to $216{ }^{\circ} \mathrm{C}$, the extraction ratio significantly increased from $10.15 \%$ to $11.31 \%$ (Budryn et al., 2012).

Effect of brewing on lipid content The amount of lipid extracted from coffee residue was evaluated in medium roasted coffee and the extraction ratio is presented in Fig. 3. The moisture content of the residue sample was $58.34 \pm 0.59 \%$, and this value was used to calculate the oil extraction ratio. The coffee residue showed an oil extraction ratio of $5.9 \%$ (d.w.), which was about $50 \%$ of the total oil content. It seems that when coffee was brewed with hot water, $50 \%$ of the oil did not rise to the surface to be eluted into the coffee liquid, but remained inside the beans. The oil extraction ratio of the coffee residue obtained in this study (5.9\%) was much lower than previously reported values (12.33-25.84\%) (Efthymiopoulos et al., 2019; Haile, 2014). This may be related to the different preprocessing procedures utilized. In the present study, hexane 


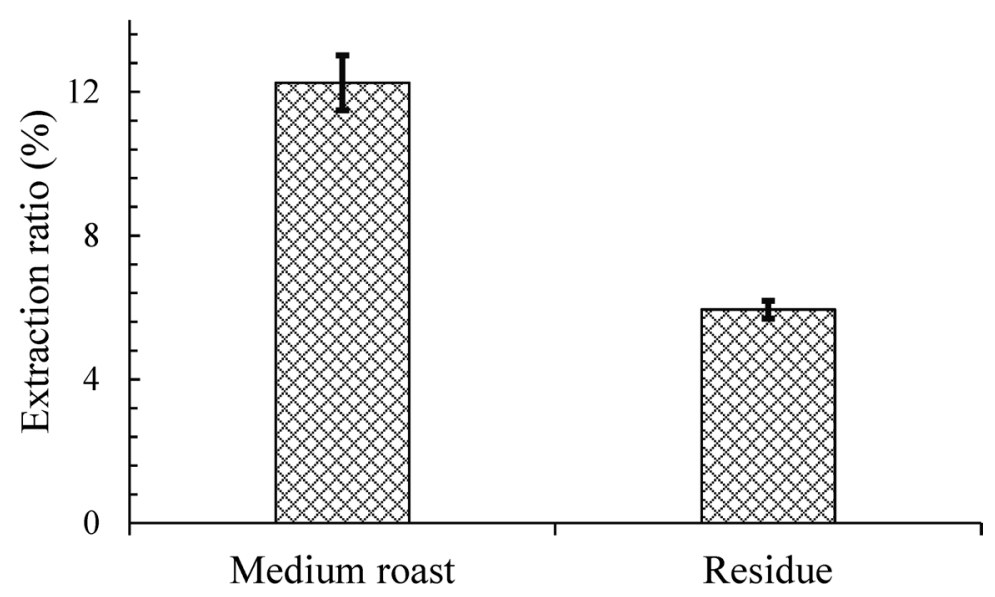

Fig. 3. Effect of brewing on oil extraction

extraction was carried out using the residue directly after brewing, whereas in previous studies, the residue was ovendried before organic solvent extraction. The difference in the oil extraction ratio is considered to be derived from the difference in surface condition (wet or dry) of the samples.

Fatty acid composition of extracted coffee oil Coffee oil extracted by the Soxhlet method showed a light brown color. Coffee oil contains active ingredients, including various aroma components and lipophilic Maillard products that exhibit strongly bitter tastes (Campos-vega and Oomah, 2015).

Out of the eleven standard fatty acids analyzed, coffee oil extracted from roasted beans exhibited eight kinds of fatty acid, while the residue oil contained seven kinds of fatty acid. Fig 4. shows representative chromatograms of the four kinds of sample. The roasted coffee oils contained the following fatty acids: myristic (C14:0), palmitic (C16:0), palmitoleic (C16:1), stearic (C18:0), oleic (C18:1), linoleic (C18:2), linolenic (C18:3), and arachidic (C20:0) acid. Eicosenoic (C20:1), behenic (C22:0), and lignoceric (C24:0) acids were not found in the roasted coffee oil. This may be due to the low content or the extraction method and conditions (Speer and KöllingSpeer, 2006). The oil extracted from the residue contained the following seven fatty acids: myristic, palmitic, stearic, oleic, linoleic, linolenic, and arachidic acid.

The eight fatty acids detected in the roasted coffee oils were quantified and their ratios are presented in Table 1 . The GC/FID chromatographic analysis of the coffee oil extract shows that the coffee oil was composed of $42.0-46.1 \%$ of saturated fatty acids (SFA) such as palmitic acid and $53.8-58.0 \%$ of unsaturated fatty acids (UFA) such as linoleic acid. Among those fatty acids, linoleic acid (45.2-47.5\%) and palmitic acid (34.6-39.1\%) showed the highest content. These results are in good agreement with those reported by Martín et al. (2001) and by Khan and Brown (1953).

UFA is further divided into monounsaturated fatty acids (MUFA) and polyunsaturated fatty acids (PUFA). The ratios of SFA, UFA, MUFA, and PUFA are shown in Table 2. The content of UFA is a critical factor for nutritional value, and
PUFA is expected to have essential health benefits (Christie and Han, 2012). MUFA content from roasted coffee oil was $7.35-9.01 \%$, and PUFA was $46.53-48.95 \%$. The findings of the present study are generally similar to the previous study carried out by Budryn et al. (2012), who reported that MUFA content from residue coffee oil was $8.31 \%$ and PUFA was $47.51 \%$. Similar results were obtained from Efthymiopoulos et al. (2019).

From the quantification of fatty acid profiles of light, medium, and dark roasted coffee oil and residue coffee oil, there was no significant difference in the fatty acid ratio between samples of different roasting conditions. However, the fatty acid composition tended to change with roasting. The content ratio of fatty acids with relatively short carbon chains (C14:0, C16:0, C16:1) were increased by roasting, and the content ratio of fatty acids with relatively long carbon chains (C18:0, C18:1, C18:2, C18:3, C20:0) were decreased by roasting.

In general, the solubility of fatty acids in water decreases as the carbon number increases. However, the absence of significant differences in the FA profiles of the four oil samples, including those of the residue coffee oil, suggests that there was little difference in the water solubility of the fatty acids identified in this study. This may be because the coffee oils extracted were composed of fatty acids with relatively long carbon chains, with carbon numbers in the range from 14 to 20 . This study indicated that coffee roasting conditions and extraction for beverage processing were not important factors for fatty acid composition. Our findings showed a good agreement with the results reported by Calligaris et al. (2009) who investigated the effect of roasting on the fatty acid composition of commercial coffee powder.

On the other hand, stearic acid, oleic acid, and arachidic acid showed a larger content ratio in the residue coffee than roasted coffee, although the results were not statistically significant. This result agrees with the result reported by Efthymiopoulos et al. (2019) who studied the physical properties of coffee residues and the characteristics of the oil. 


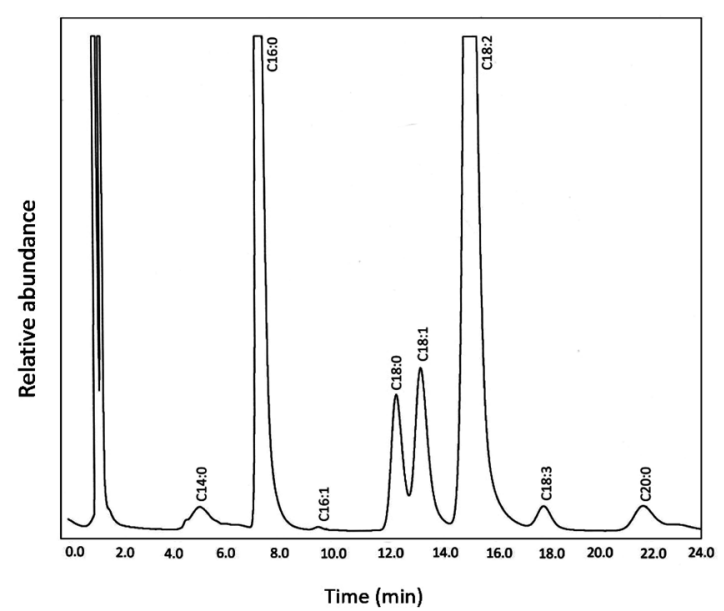

(a) Dark roast

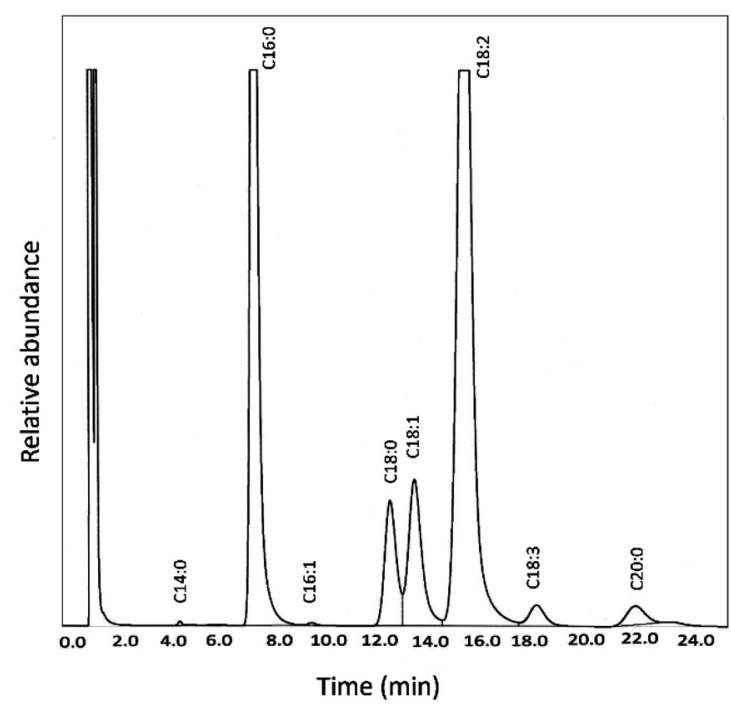

(c) Light roast

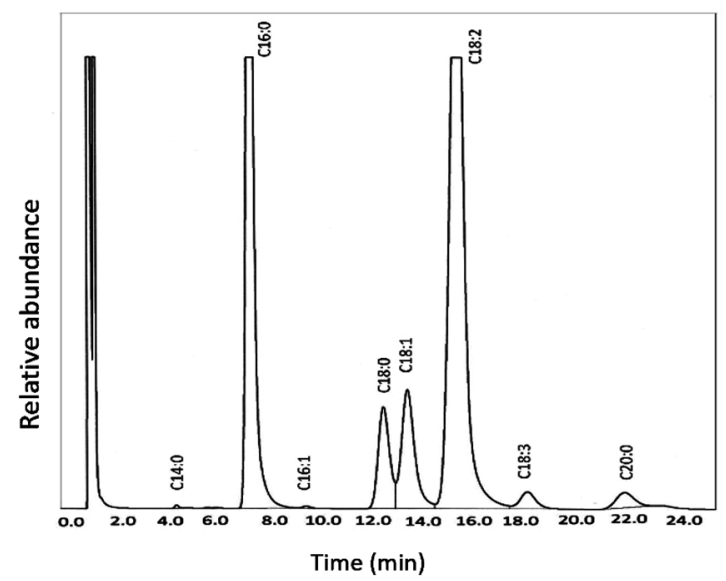

(b) Medium roast

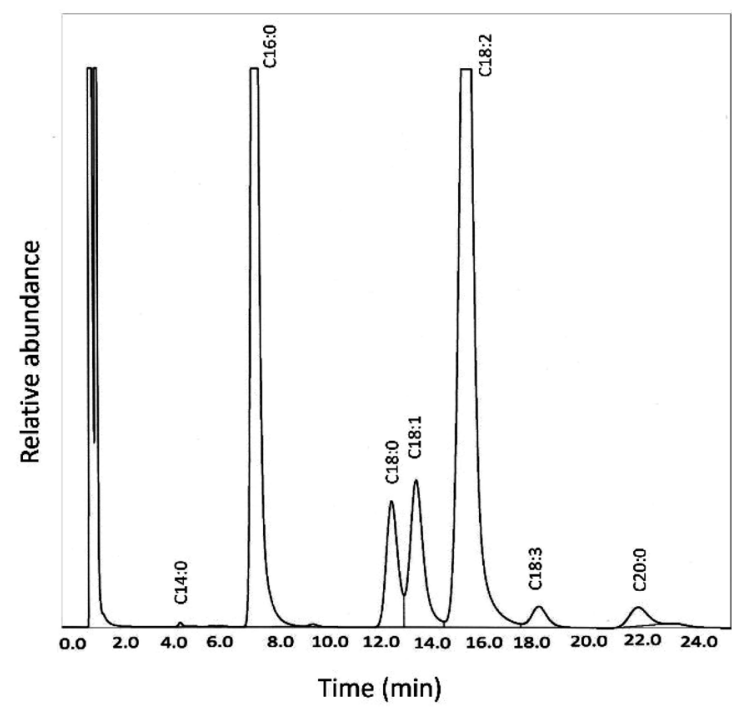

(d) Residue

Fig. 4. Typical chromatogram of fatty acid of extracted oil from coffee bean and residue

Table 1. Composition of fatty acids of extracted coffee oil (\%)

\begin{tabular}{lcccc}
\hline \multirow{2}{*}{ Fatty acids } & \multicolumn{4}{c}{ Content ratio (\%) } \\
\cline { 2 - 5 } & Light roast & Medium roast & Dark roast & Residue \\
\cline { 2 - 5 } Myristic acid & 0.06 & 0.06 & 0.08 & 0.07 \\
Palmitic acid & 34.56 & 34.83 & 39.14 & 36.00 \\
Palmitoleic acid & 0.29 & 0.08 & 0.07 & N.D. \\
Stearic acid & 6.01 & 6.16 & 5.57 & 6.47 \\
Oleic acid & 8.71 & 8.49 & 7.28 & 8.31 \\
Linoleic acid & 47.53 & 47.54 & 45.15 & 46.47 \\
Linolenic acid & 1.42 & 1.41 & 1.39 & 1.03 \\
Arachidic acid & 1.42 & 1.42 & 1.33 & 1.63 \\
\hline
\end{tabular}

N.D. $=$ Not detected 
Table 2. Composition of saturated fatty acids (SFA) and unsaturated fatty acids (UFA) of extracted coffee oil (\%)

\begin{tabular}{lcccc}
\hline & \multicolumn{4}{c}{ Content ratio (\%) } \\
\cline { 2 - 5 } & Light roast & Medium roast & Dark roast & Residue \\
\cline { 2 - 5 } SFA & 42.04 & 42.48 & 46.11 & 44.18 \\
UFA & 57.96 & 57.52 & 53.89 & 55.82 \\
MUFA & 9.01 & 8.57 & 7.35 & 8.31 \\
PUFA & 48.95 & 48.95 & 46.53 & 47.51 \\
\hline
\end{tabular}

$\mathrm{SFA}=$ Saturated fatty acid, UFA $=$ Unsaturated fatty acid, MUFA $=$ Monounsaturated fatty acid,

PUFA $=$ Polyunsaturated fatty acid

\section{Conclusion}

Coffee oil was extracted from different kinds of roasted coffee and coffee residue. The roasted coffee oil contained eight kinds of fatty acids, and the oil extracted from coffee residue contained seven kinds of fatty acids. The major fatty acids were: myristic (C14:0), palmitic (C16:0), stearic (C18:0), oleic (C18:1), linoleic (C18:2), linolenic (C18:3), and arachidic (C20:0) acids. Palmitoleic (C16:1) acid was detected only in the roasted coffee oil. The fatty acid compositions were not significantly affected by roasting and brewing; however, the total amount differed significantly. As much as $50 \%$ of the total oil remained in the residue of brewed coffee, and the remaining oil is expected to contain low volatile aroma components. According to the results obtained in this study, the fatty acid composition of coffee oil was not affected by heat during roasting and hot water extraction. Therefore the coffee residue is resource-rich in coffee oil, and this oil has a potential to be utilized widely for food flavoring in low-quality coffee, confectioneries, and dairy products. The effective use of coffee oil in the residue could provide economic and environmental benefits.

Acknowledgments The authors thank SAZA COFFEE 1td., Co for providing the coffee bean samples and for valuable advice on coffee roasting.

\section{References}

Alves, M.R., Casal, S., Oliveira, M.B.P.P., and Ferreira, M.A. (2003). Contribution of FA profile obtained by high-resolution GC/ chemometric techniques to the authenticity of green and roasted coffee varieties. Journal of the American Oil Chemists Society, 80, 511-517.

Budryn, G., Nebesny, E., Zyzelewicz, D., Oracz, J., Miśkiewicz, K., and Rosicka-Kaczmarek, J. (2012). Influence of roasting conditions on fatty acids and oxidative changes of Robusta coffee oil. European Journal of Lipid Science and Technology, 114, 1052-1061.

Calligaris, S., Munari, M., Arrighetti, G., and Barba, L. (2009). Insights into the physicochemical properties of coffee oil. Eur. J. Lipid Sci. Technol., 1270-1277.

Campos-vega, R. and Oomah, B.D. (2015). Spent coffee grounds : A review on current research and future prospects. Trends in Food Science and Technology, 45, 24-36.

Christie, W.W. and Han, X. (2012). Lipid analysis: isolation, separation, identification and lipidomic analysis Fourth edition. Philadelphia, PA, USA: Woodhead Publishing.

Efthymiopoulos, I., Hellier, P., Ladommatos, N., Kay, A., and Lamptey, B.M. (2019). Effect of solvent extraction parameters on the recovery of oil from spent coffee grounds for biofuel Production. Waste and Biomass Valorization, 10, 253-264.

Giacalone, D., Kreuzfeldt, T., Yang, N., Liu, C., Fisk, I., and Münchow, M. (2019). Common roasting defects in coffee : Aroma composition, sensory characterization and consumer perception. Food Quality and Preference, 71, 463-474.

Haile, M. (2014). Integrated volarization of spent coffee grounds to biofuels. Biofuel Res. J., 2, 65-69.

Jokanović, M.R., Džinić, N.R., Cvetković, B.R., Grujić, S., and Odžaković, B. (2012). Changes of physical properties of coffee beans during roasting. Acta Periodica Technologica, 43, 21-31.

Khan, N.A. and Brown, J.B. (1953). The composition of coffee oil and its component fatty acids. Journal of the American Oil Chemists Society, 30, 606-609.

Martín, M.J., Pablos, F., González, A.G., Valdenebro, M.S., and LeónCamacho, M. (2001). Fatty acid profiles as discriminant parameters for coffee varieties differentiation. Talanta, 54, 291-297.

Maruo, S. and Hirose, Y. (2009). Kohi no koumi wo saguru + Humi hyougen yougo shu (Exproring the flavor of coffee + Glossary of flavor expressions). Asahiya shuppan Ltd.

Microbiol, B., Biotecnologicos, P., Quimica, E., Federal, U., and Fruiting, C. (2000). Solid state cultivation -an efficient method to use toxic agro-industrial residues, 40, 187-197.

Mussatto, S.I., Carneiro, L.M., Silva, J.P.A., Roberto, I.C., and Teixeira, J.A. (2011a). A study on chemical constituents and sugars extraction from spent coffee grounds. Carbohydrate Polymers, 83, 368-374.

Mussatto, S.I., Machado, E.M.S., Martins, S., and Teixeira, J.A. (2011b). Production, composition, and application of coffee and its industrial residues. Food Bioprocess Technology, 661-672.

Oliveira, A.L. De, Cruz, P.M., Eberlin, M.N., and Cabral, F. a. (2005). Brazilian roasted coffee oil obtained by mechanical expelling: compositional analysis by GC-MS. Ciência e Tecnologia de 
Alimentos, 25, 677-682.

R.J. Clarke and Macrae, R. (1985). COFFEE Volume 1 CHEMISTRY. London and New York: Elsevier.

Ratnayake, W.M.N., Hollywood, R., O'Grady, E., and Stavric, B. (1993). Lipid content and composition of coffee brews prepared by different methods. Food and Chemical Toxicology, 31, 263-269.

Speer, K. and Kölling-Speer, I. (2006). The lipid fraction of the coffee bean. Brazilian Journal of Plant Physiology, 18, 201-216.

Tanbe, Y. (2016). Kohi no kagaku (Science of coffee). Japan, Tokyo: Kodansha Ltd.

Yovana, M., Jesus, T. De, Salva, G., and Bragagnolo, N. (2014). Impact of chemical changes on the sensory characteristics of coffee beans during storage. Food Chem., 147, 279-286. 